Widefield ScIENCE AND TeChNOLOGY For the SKA

SKADS CONFERENCE 2009

S.A. Torchinsky, A. van Ardenne, T. van den Brink-Havinga, A.J.J. van Es, A.J. Faulkner (eds.)

4-6 November 2009, Château de Limelette, Belgium

\title{
The SKA AA-lo array; E.M. simulation and design *
}

\author{
E. de Lera Acedo ${ }^{1}$, N. Razavi-Ghods ${ }^{1}$, D. González-Ovejero ${ }^{2}$, E. García ${ }^{3}$, C. Craeye ${ }^{2}$, P. J. Duffett-Smith ${ }^{1}$ and \\ P. Alexander ${ }^{1}$
}

1 Cavendish Laboratory, University of Cambridge, CB3 OHE, Cambridge UK

${ }^{2}$ Laboratoire de Télécommunication et Télédétection, Université Catholique de Louvain, 1348 Louvain-la-Neuve, Belgium

3 Signal Theory and Communications Department, University Carlos III of Madrid, Leganes, Madrid, 28911 Spain

\begin{abstract}
The SKA relies on the use of very large phased arrays. Furthermore, the SKA community is considering the use of non-periodic sparse configurations instead of the classical regular arrays to cover the sub $500 \mathrm{MHz}$ band. The cost in the design process for an irregular array is evident, as now periodic infinite array approaches, available in many commercial simulation packages, are no longer applicable. The method proposed by the authors to reduce the computational cost associated to solve the full EM problem in such arrays is based in a Macro Basis Function (MBF) technique and an interpolation method. The embedded element patterns for each antenna are computed by assuming interactions between only a few tens of elements. This approximation represents a first step towards a very fast and cost-effective full-wave simulation tool for very large non-periodic arrays. The authors also show why a proper full-wave simulation of a non-periodic array is necessary. The main idea behind it is that the effects of mutual coupling are not fully randomized out for some practical realizations of the array. Also, some relevant aspects about regular sparse arrays are analyzed and presented in terms of the effective aperture and the system noise temperature of the unit cell.
\end{abstract}

\section{Introduction}

The current frequency range of the SKA will be made possible using multiple collector technologies, consisting mainly of dishes at high frequencies $(>1 \mathrm{GHz})$ and aperture arrays for the key science of detecting red-shifted hydrogen emission at around $1 \mathrm{GHz}$ and below. One particular science objective of the SKA is to study the formation of the first galaxies which resulted in the cold neutral hydrogen pervading much of the universe at this time being ionized. This so-called "epoch of re-ionisation" occurs at a sufficiently early time that the signals from the 21-cm line of neutral hydrogen are "redshifted" into the frequency range from $70 \mathrm{MHz}$ through to several hundred $\mathrm{MHz}$. One part of the SKA design therefore calls for an ultrawide band antenna array able to operate from 70-450 MHz. This is called the SKA AA-lo. At these low frequencies the telescope can provide a million square meters of collecting area, which is two orders of magnitude more sensitivity than any current instrument. The SKA AA-lo will comprise up to hundreds of stations of at least 10000 elements each to meet the sensitivity requirements $\left(4000 \mathrm{~m}^{2} / \mathrm{K}\right.$ at $\left.100 \mathrm{MHz}\right)$. There are clear cost saving if this frequency range can be covered by a single antenna technology. Visit www.skatelescope.org for more information.

Sparse array configurations are of interest for the SKA because of its larger effective aperture compared to closelypacked arrays (Braun et al. 2006), where the effective aperture is limited by the physical space allocated to each antenna in the array. This is especially important at low frequencies $(<$

\footnotetext{
^ This work was supported by the European Commission Framework Program 6, Project SKADS, Square Kilometre Array Design Studies (SKADS), contract no 011938.
}

$200 \mathrm{MHz}$ ), as the sky brightness incrases exponentially for decreasing frequencies (Cortes Medellin 2007).

Irregular array configurations are also of interest for the SKA. For example, by randomizing the position of the antennas in the array grid, localized undesired effects, such as grat ing lobes, may be spread over the parameter space. This will increase the noise ground of the telescope but will avoid blind angles or localized malfunctions of the instrument. It is yet to be decided which is more convinient for the SKA. Narrow band anomalies caused by mutual coupling are other example of undesired effects which could be mitigated by the use of nonperiodic arrays. Both, sparse and non-periodic array configurations are the focus of this paper.

Unfortunately, it is not possible at present to do a full-wave simulation of a real size SKA stations (up to at least 10000 elements). Gonzalez-Ovejero \& Craeye (2008) proposed a method for fast computation of the interactions between MBFs based in a sub-gridding technique. This work has now been extended (Gonzalez-Ovejero et al. 2009) in order to simulate a real size SKA station.

The paper is structured as follows. In Section 2 the method proposed by the authors to reduce the computational cost devoted to solve the full EM problem in irregular arrays is presented. Furthermore, the necessity of this type of tools is shown by analyzing the errors in a nulling application when mutual coupling effects are dismissed. Section 3 focuses on the design study of an ultra wide-band bow-tie antenna element in an aperture array environment, emphasizing those characteristics important for the SKA. We draw some conclusions in Section 4. 


\section{Method of Moments simulation of large irregular arrays}

The proposed Method of Moments (MoM) simulation for this problem relies on the use of Macro Basis Functions (MBFs) (Suter \& Mosig 2000; Craeye 2006; Craeye \& Sarkis 2008), also called Characteristic Basis Functions (Yeo et al. 2003; Maaskant et al. 2008), and the interpolation technique presented in Gonzalez-Ovejero \& Craeye (2008). The Macro Basis Functions method consists of reducing the size of the MoM impedance matrix by replacing the original set of elementary basis functions with a new set of functions obtained through the solution of smaller problems. Once the MoM matrix size is reduced by means of the MBF technique, the complexity $O\left((N A)^{3}\right)$ for solving the system of equations is reduced to $O\left((Q A)^{3}\right)$, where $A$ is the number of antennas in the array and $N$ and $Q(Q<<N)$ are the number of elementary and Macro Basis Functions, respectively. Nevertheless, the impedance matrix filling time remains $O\left((N A)^{2}\right)$, and rapidly becomes the dominant operation in the total solution time. To overcome this limitation, the computation of interactions between Macro Basis Functions is carried out by interpolating exact data obtained on a simple grid; thereby non regular arrays may be easily analyzed as well as optimized. The complexity will be now $O\left(P N^{2}+A^{2} S\right.$ ), where $P$ (typically around some tens or very few hundreds) is the number of elements in the grid and $S$ is a very small factor related to the interpolation time. It is interesting to notice that this factor no longer depends on the complexity of the antenna. Therefore a speed up of the order of $A$ can be obtained.

The initial target is to obtain the pattern for an example comprising 1000 bowtie elements, like the one shown in Fig. 1a, randomly placed in a circular area of radius $30 \lambda$ (Fig. 1b), which at this point would represent a reduced version of the aforementioned SKA AA-lo. For the present analysis the average minimum distance between elements is $1.5 \lambda$ and the elements size is $\lambda \times \lambda$. Each port has been loaded with a $200 \Omega$ series impedance.

A so-called radius of influence, can be defined for every antenna in the array. The embedded pattern can then be computed accounting only for the antennas contained in the circle defined by this radius. Assuming that the latter approximation is good enough, the complexity in the calculation of the interactions and the solution of the MoM system of equations can be dramatically reduced. The system can be solved for each antenna or for the whole array at once, which by making use of sparse matrices can reduce further the computation time.

The convergence of the method is proven in GonzalezOvejero et al. (2009). Once we have the embedded element pattern for each antenna, in order to obtain the total pattern for the proposed array, all the embedded element patterns are combined through the excitation law of interest. This is shown in Fig. 2a and Fig. 2b. Also in Fig. 2a and Fig. 2b the array radiation pattern computed from the single element patterns (dismissing mutual coupling effects) is shown. Despite the apparent similarity between both approaches there is a systematic bias between them. This bias must be taken into account in the

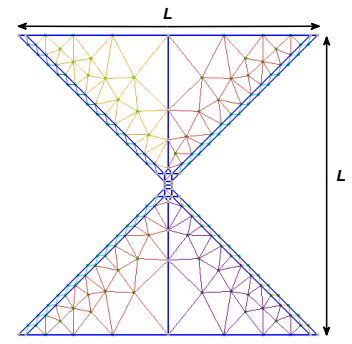

Fig. 1a: Bow-tie antenna element of size $L \times L$. The element is placed $\lambda / 4$ on top of the ground plane.

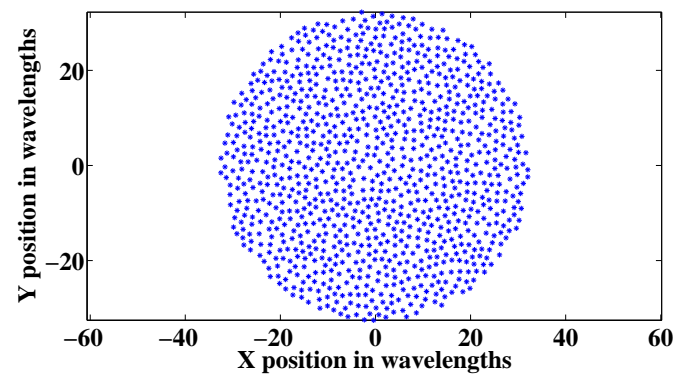

Fig. 1b: Array layout

design of the SKA AA-lo as it might affect the performance of the array dramatically.

Fully random arrays are not achivable in principle due to the finite size of the arrays and the non-zero size of each antenna element. Depending on the degree of radomization of the antenna positions, the mutual coupling effects are also likely to be randomized out. However, non-periodic grids will still suffer from mutual coupling and its effects won't cancel out. For example, when doing certain applications, such as nulling, the forced null can be affected if the weights have been computed without accounting for mutual coupling. In Fig. 3 one can observe this effect in a simulation where the array pattern has been computed for the same angles in both cases. It is now apparent how the coupling effects are not fully randomized out for a non-periodic array with a high randomness degree. Therefore, numerical methods capable of simulating large irregular arrays of sizes up to hundreds of wavelengths are needed.

The reduced impedance matrix filling time was 77 minutes when using MBFs and 20 seconds for the interpolation technique (without accounting for the grid generation time) in a standard laptop.

\section{Sparse arrays for the AA-lo band}

The most important figure of merit for low frequency radio astronomy instruments is the system sensitivity, defined as $A_{e f f} / T_{s y s}$ (Eq. (22) in Ivashina et al. 2008). In the present study, $A_{e f f}$ is obtained by simulating the Gain of a unit cell of the infinite regular array, $G_{c e l l}$, when this is phased to point in a given direction $r_{0}=\left(\theta_{0}, \phi_{0}\right)$. (Each unit cell contains a single bow-tie antenna element as in Fig 1a.) The system tem- 


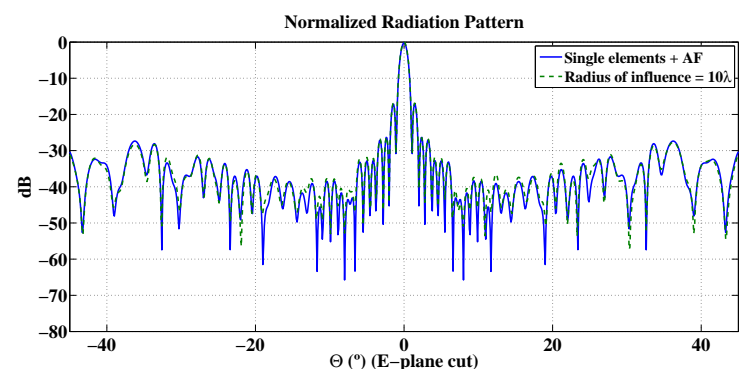

Fig. 2a: E-plane cut

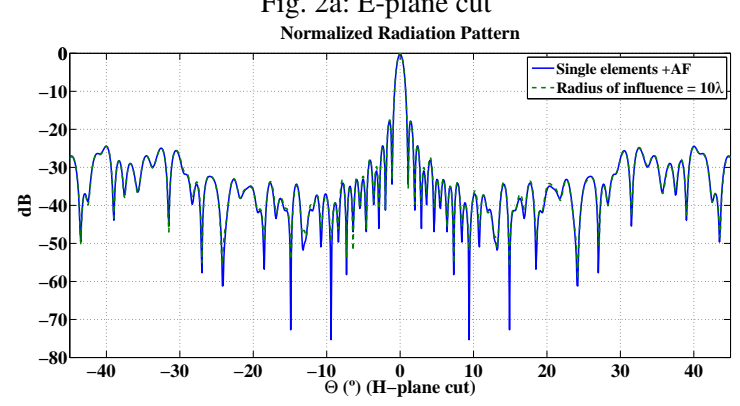

Fig. 2b: H-plane cut

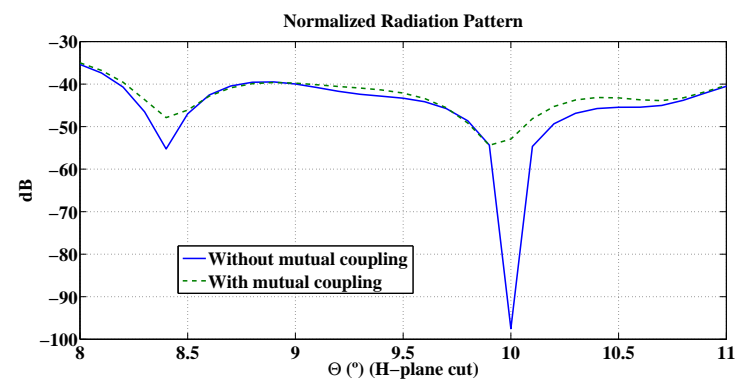

Fig. 3: H-plane cut of the array pattern when a null is forced at $10^{\circ}$ using the single element patterns

perature, $T_{s y s}$, as described in de Lera Acedo et al. (2009), accounts for the contributions from the sky brightness, losses suffered before the amplifier, and electronic noise generated by the amplifier. These are also dependant on $r_{0}$. Therefore, the selection of an antenna element for the SKA AA-lo, should be strongly based on these 2 parameters.

An infinite regular phased array of the proposed elements has been simulated with the periodic boundaries approach of commercial software based on FD-TD, CST (www.cst.com). The element is made of Perfect Electric Conductor (PEC) and it is placed a quarter of a wavelength at the highest frequency of interest (166 mm at $450 \mathrm{MHz}$ ) above a ground plane to optimize the beam shape of the element. The use of a ground plane avoids uncontrolled and lossy reflections in the actual ground and makes the element sensitive only to the radiation coming from the sky. However, it is important to notice that the wideband nature of the antenna will now be limited by the presence of the ground plane. The main effect is that the impedance at the low end of the frequency band is reduced. Fortunately, the pres- ence of the sky noise allows the designer to relax the matching constrain at those low frequencies without losing much sensitivity.

Fig. 4 shows the effective aperture of a unit cell when the array is pointing to broadside for different inter-element spacings. One can see 2 regions in this plot. A first region up to those frequencies at which the inter-element spacing, $d$, is $\lambda$. In this lower frequency band the effective aperture is approximately constant with frequency and grows with $d$. However, the upper frequency band, for inter-element spacings greater than $\lambda$, shows an effective aperture decreasing with increasing frequency at an approximate rate equal to $\lambda^{2} / 2$ for all $d$. This effect has already been reported before for an array of dipoles by Braun et al. (2006). These regions represent the dense and sparse regimes of the array. In actual fact for the present case it seems that in the sparse regime, the effective aperture is slightly better than $\lambda^{2} / 2$. In the dense regime the effective aperture of the unit cell converges to its physical size, $d^{2}$, as expected. Furthermore, the efficiency of the unit cell improves for more packed configurations. This is due to the more uniform illumi $\downarrow$ nation of smaller unit cells. It is important to note that even with a relatively small bow-tie element, it is still possible to achieve a good effective aperture for the required frequency band.

For different element sizes, as shown in Fig. 5, the effective aperture of the unit cell reaches a maximum value equal to the physical area of the unit cell in the dense regime, almost independently of the size of the element. Furthermore, larger antennas are likely to produce multi-lobulation effects, which may limit the usable frequency band.

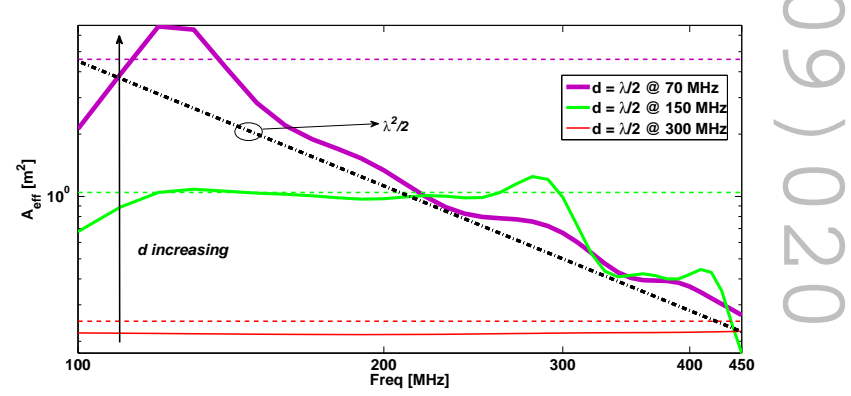

Fig. 4: Effective aperture for different inter-element spacings, $d$. The dashed lines represent the physical size of the unit cell

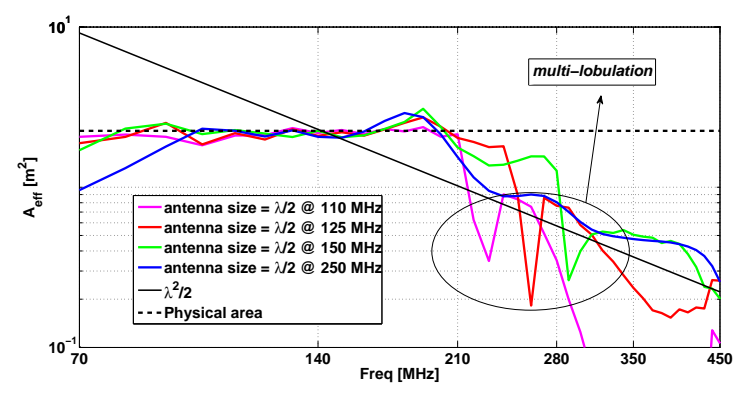

Fig. 5: Effective aperture for different antenna sizes, $L$ 
The sparseness ratio of the array and the antenna size also have an impact on the $T_{\text {sys }}$. Fig. 6 shows how by placing elements closer to each other in the array layout, the beneficial effects of mutual coupling may improve the impedance of the antenna elements and therefore the matching to the LNA. Ultimately, this reduces the system noise, composed not only of the sky brightness, but of a receiver noise factor which may determine the performance of the array (see de Lera Acedo et al. 2009). Also, a better $T_{\text {sys }}$ can be achieved at low frequencies by increasing the size of the elements, as it could be expected.

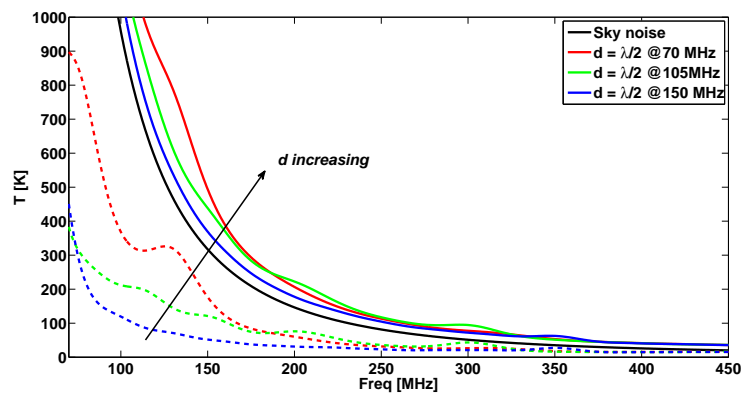

Fig. 6: System temperature for different inter-element spacings, $d$. The dashed lines represent the receiver noise

\section{Conclusions}

This paper summarizes the efforts of the authors to develop a full-wave simulation tool based on MoM and an interpolation technique. The authors have also proved the necessity of these type of softwares for large irregular arrays such as the SKA AA-lo.

An analytical study has been described here of a bow-tie antenna element immersed in an infinite regular sparse array, as a potential candidate for the SKA AA-lo, and it both refutes some well known facts about antenna theory and gives designers some useful hints on how to design a radio astronomy station. Larger elements, with sizes comparable to the wavelength, are likely to give better sensitivities at low frequencies because of better matching to the amplifier, but at the cost of a more expensive antenna. However, the worse system temperature delivered by a smaller element can be partially mitigated by taking advantage of mutual coupling and placing antennas in a denser configuration. Sparser arrays deliver better effective aperture over the whole band. Also, a trade-off is necessary when finding the optimum size of the antenna for such an ultra wideband application, as very large antenna elements display multiple lobes.

An optimized version of the proposed bow-tie antenna element, the BLU antenna (Bow-tie Low-frequency Ultra wideband antenna) has been built and is ready for measurement (see Fig. 7). Furthermore, a 400-element scaled version of an SKA AA-lo station is being designed and will be soon built to prove the concept of the bow-tie elements and test different array configurations.

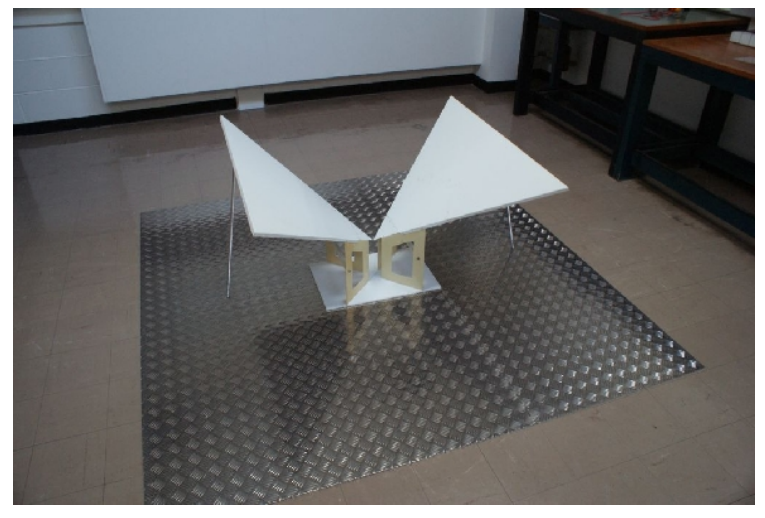

Fig. 7: The BLU antenna

Acknowledgements. We are grateful to our SKA colleagues for several discussions around this topic.

\section{References}

Braun, R. \& van Cappellen, W., SKA Memo 87: "aperture arrays for the SKA: dense or sparse?" Available online: WWw . skatelescope.org/PDF/memos/memo_87.pdf

Cortes Medellin, G., SKA Memo 95: Antenna Noise Temperature Calculation. Available online: wWW . skatelescope.org/PDF/memos/Memo_95.pdf

Craeye, C., A fast impedance and pattern computation scheme for finite antenna arrays, in IEEE Trans. Antennas Propag., vol. 54, pp. 3030-3034, October 2006

Craeye, C., \& R. Sarkis, Finite array analysis through combination of Macro Basis Functions and Array Scanning Methods, in Journal of Applied Comput. Electromagnetics Soc. (ACES), vol. 23, pp. 999-1007, September 2008

de Lera Acedo, E., N. Razavi-Ghods, E. Garcia, P. J. Duffett-Smith \& Paul Alexander, System Noise Analysis of an Ultra Wide Band Aperture Array Element for Low Frequency Radio Astronomy, in Proceedings of the 6th IASETD International Conference Antennas, Radar, and Wave Propoagation (ARP 2009), Banff, Alberta, Canada, July 6-8, 2009

Gonzalez-Ovejero, D., \& C. Craeye, Fast computation of Macro Basis Functions interactions in non-uniform arrays, in Proc. IEEE AP-S Soc. Int. Symp., San Diego, CA, July 2008

Gonzalez-Ovejero, D., E. de Lera Acedo, N. Razavi-Ghods, \& C. Craeye, Fast MBF based method for large random array characterization, in IEEE Antennas and Propagation Society International Symposium, APS-URSI'09, 1-5 June 2009, pp. $1-4$.

Ivashina, M., R. Maaskant, \& B. Woestenburg, Equivalent system representation to model the beam sensitivity of receiving antenna arrays, in Antennas and Wireless Propagation Letters, IEEE, Vol. 7, pp: 733 737, 2008

Maaskant, R., R. Mittra, \& A. Tijhuis, Fast analysis of large antenna arrays using the Characteristic Basis FunctionMethod and the Adaptive Cross Approximation Algorithm, in IEEE 
Trans. Antennas Propag., vol. 56, pp. 3440-3451, November 2008

Suter, E., \& J. R. Mosig, "A subdomain multilevel approach for the efficient MoM analysis of large planar antennas," in Microw. Opt. Technol. Lett., vol. 26, pp. 270-277, March 2000

Yeo, J., V. Prakash, \& R. Mittra, Efficient analysis of a class of microstrip antennas using the Characteristic Basis Function Method (CBFM), in Microw. Opt. Technol. Lett., vol. 39, pp. 456-464, December 2003 